\title{
ARITHMETIC MEAN OF VALUES AND VALUE AT MEAN OF ARGUMENTS FOR CONVEX FUNCTIONS
}

\author{
CRISTINEL MORTICI ${ }^{1}$
}

(Received 19 February, 2007; revised 3 September, 2008)

\begin{abstract}
We give here some extensions of inequalities of Popoviciu and Rado. The idea is to use an inequality [C. P. Niculescu and L. E. Persson, Convex functions. Basic theory and applications (Universitaria Press, Craiova, 2003), Page 4] which gives an approximation of the arithmetic mean of $n$ values of a given convex function in terms of the value at the arithmetic mean of the arguments. We also give more general forms of this inequality by replacing the arithmetic mean with others. Finally we use these inequalities to establish similar inequalities of Popoviciu and Rado type.
\end{abstract}

2000 Mathematics subject classification: 26D15, 52A40.

Keywords and phrases: convex functions, means, Popoviciu's inequality, Rado's inequality.

\section{Introduction}

For $x_{1}, \ldots, x_{k}>0, k \geq 1$ we put

$$
A_{k}=\frac{x_{1}+\cdots+x_{k}}{k}, \quad G_{k}\left(x_{1} \ldots x_{k}\right)^{1 / k} .
$$

Inequalities involving the arithmetic mean and the geometric mean are of great interest. We discuss here the inequality of Popoviciu

$$
\left(\frac{A_{n}}{G_{n}}\right)^{n} \geq\left(\frac{A_{n-1}}{G_{n-1}}\right)^{n-1} \geq \cdots \geq\left(\frac{A_{1}}{G_{1}}\right)^{1}=1
$$

and the inequality of Rado

$$
n\left(A_{n}-G_{n}\right) \geq(n-1)\left(A_{n-1}-G_{n-1}\right) \geq \cdots \geq 1 \cdot\left(A_{1}-G_{1}\right)=0 .
$$

\footnotetext{
${ }^{1}$ Department of Mathematics, Valahia University of Targoviste, Bd. Unirii 18, Targoviste, Romania; e-mail: cmortici@valahia.ro.

(C) Australian Mathematical Society 2008, Serial-fee code 1446-1811/08 $\$ 16.00$
} 
For proofs and other consequences, see [1]. We prove that the inequality (1.1) remains true if we replace $A_{k}$ with

$$
A_{k}^{p}=\left(\frac{x_{1}^{p}+\cdots+x_{k}^{p}}{k}\right)^{1 / p}, \quad p \geq 1 .
$$

Moreover, we prove that (1.2) holds also if we replace the geometric means $G_{k}$ with other means.

One way to attack these inequalities is to use a nice inequality related to convex functions (for example [2, Page 4]) denoted here by (1.3).

Let $I \subseteq \mathbb{R}$ be an interval. We say that a function $f: I \rightarrow \mathbb{R}$ is convex if for all $x, y \in I$ and for each $\alpha \in[0,1]$,

$$
f(\alpha x+(1-\alpha) y) \leq \alpha f(x)+(1-\alpha) f(y) .
$$

The function $f$ is called concave if the above inequality holds with reverse sense.

If $f$ is convex, then for

$$
\alpha=\frac{n-1}{n}, \quad x=\frac{x_{1}+\cdots+x_{n-1}}{n-1}, \quad y=x_{n}
$$

we deduce that

$$
f\left(\frac{x_{1}+\cdots+x_{n}}{n}\right) \leq \frac{1}{n} f\left(x_{n}\right)+\frac{n-1}{n} f\left(\frac{x_{1}+\cdots+x_{n-1}}{n-1}\right) .
$$

Therefore

$$
\begin{aligned}
& n\left[\frac{f\left(x_{1}\right)+\cdots+f\left(x_{n}\right)}{n}-f\left(\frac{x_{1}+\cdots+x_{n}}{n}\right)\right] \\
& \quad \geq(n-1)\left[\frac{f\left(x_{1}\right)+\cdots+f\left(x_{n-1}\right)}{n-1}-f\left(\frac{x_{1}+\cdots+x_{n-1}}{n-1}\right)\right]
\end{aligned}
$$

In other words, the sequence

$$
a_{n}=n\left[\frac{f\left(x_{1}\right)+\cdots+f\left(x_{n}\right)}{n}-f\left(\frac{x_{1}+\cdots+x_{n}}{n}\right)\right], \quad n \geq 1
$$

is monotonically increasing, where $\left(x_{n}\right)_{n \geq 1} \subset I$ is arbitrarily given.

The inequalities (1.1)-(1.2) follow now by applying (1.3) to $f(x)=-\ln x$ and $f(x)=e^{x}$ respectively.

\section{The results}

We give some general results concerning the sequence (1.4), then we study some particular cases to obtain results of the form (1.1) and (1.2). We use the following well-known result. 
LEMMA 2.1. Let $I, J \subseteq \mathbb{R}$ be intervals. Assume that $\phi: I \rightarrow J$ is convex and $g: J \rightarrow \mathbb{R}$ is convex and monotonically increasing. Then $g \circ \phi: I \rightarrow \mathbb{R}$ is convex.

Then we have the following result.

THEOREM 2.2. Let $g: I \subseteq(0, \infty) \rightarrow \mathbb{R}$ be convex and increasing and let $\left(x_{n}\right)_{n \geq 1}$ $\subseteq(0, \infty)$. Then for every $p \in(-\infty, 1] \backslash\{0\}$, the sequence

$$
a_{n}=n\left[\frac{g\left(x_{1}\right)+\cdots+g\left(x_{n}\right)}{n}-g\left(\left(\frac{x_{1}^{p}+\cdots+x_{n}^{p}}{n}\right)^{1 / p}\right)\right], \quad n \geq 1
$$

is increasing.

PROOF. According to Lemma 2.1, the function $f(x)=g\left(x^{1 / p}\right), x>0$ is convex, because $\phi(x)=x^{1 / p}$ is convex for $p \in(-\infty, 1] \backslash\{0\}$. Thus (1.4) becomes

$$
a_{n}=n\left[\frac{g\left(x_{1}^{1 / p}\right)+\cdots+g\left(x_{n}^{1 / p}\right)}{n}-g\left(\left(\frac{x_{1}+\cdots+x_{n}}{n}\right)^{1 / p}\right)\right]
$$

and the conclusion follows by replacing $x_{k}$ with $x_{k}^{p}$.

An interesting case is $g(x)=x$ and $p=-1$ in Theorem 2.2. The respective sequence becomes

$$
a_{n}=n\left[\frac{x_{1}+\cdots+x_{n}}{n}-\left(\frac{x_{1}^{-1}+\cdots+x_{n}^{-1}}{n}\right)^{-1}\right] .
$$

Now, using the monotony of this sequence, we obtain the inequality

$$
n\left(A_{n}-H_{n}\right) \geq(n-1)\left(A_{n-1}-H_{n-1}\right) \geq \cdots \geq 1 \cdot\left(A_{1}-H_{1}\right)=0,
$$

where $A_{k}$ and $H_{k}$ are the arithmetic and harmonic means respectively:

$$
A_{k}=\frac{x_{1}+\cdots+x_{k}}{k}, \quad H_{k}=\frac{k}{1 / x_{1}+\cdots+1 / x_{k}}, \quad 1 \leq k \leq n .
$$

For $p \in(-\infty, 1] \backslash\{0\}$ and $y_{1}, \ldots, y_{k}>1$, consider the mean,

$$
W_{k}=\exp \left[\left(\frac{\ln ^{p} y_{1}+\cdots+\ln ^{p} y_{k}}{k}\right)^{1 / p}\right],
$$

to obtain the following result.

COROLlary 2.3. For any sequence $\left(y_{n}\right)_{n \geq 1} \subset(0, \infty)$,

$$
n\left(A_{n}-W_{n}\right) \geq(n-1)\left(A_{n-1}-W_{n-1}\right) \geq \cdots \geq 1 \cdot\left(A_{1}-W_{1}\right)=0 .
$$

PROOF. We apply Theorem 2.2 to $g(x)=e^{x}$. Thus the sequence

$$
a_{n}=n\left[\frac{e^{x_{1}}+\cdots+e^{x_{n}}}{n}-\exp \left(\frac{x_{1}^{p}+\cdots+x_{n}^{p}}{n}\right)^{1 / p}\right], \quad n \geq 1
$$

is increasing. The result follows by writing $e^{x_{k}}=y_{k}, 1 \leq k \leq n$. 
For $y_{1}, \ldots, y_{k}>1$ write

$$
V_{k}=\exp \left[\left(\ln y_{1} \cdots \cdot \ln y_{k}\right)^{1 / k}\right]
$$

to give the following result.

COROLlaRY 2.4. For any sequence $\left(y_{n}\right)_{n \geq 1} \subset(1, \infty)$,

$$
n\left(A_{n}-V_{n}\right) \geq(n-1)\left(A_{n-1}-V_{n-1}\right) \geq \cdots \geq 1 \cdot\left(A_{1}-V_{1}\right)=0 .
$$

PROOF. The result follows from (2.1) by taking the limit as $p \rightarrow 0$. Indeed, it is known that

$$
\lim _{p \rightarrow 0}\left(\frac{\alpha_{1}^{p}+\cdots+\alpha_{n}^{p}}{n}\right)^{1 / p}=\left(\alpha_{1} \cdots \alpha_{n}\right)^{1 / n}
$$

so $\lim _{p \rightarrow 0} W_{k}=V_{k}$, for all $1 \leq k \leq n$.

Further we use the following well-known result.

Lemma 2.5. Let $I, J \subseteq \mathbb{R}$ be intervals. Assume that $\phi: I \rightarrow J$ is concave and $g: J \rightarrow \mathbb{R}$ is convex and monotonically decreasing. Then $g \circ \phi: I \rightarrow \mathbb{R}$ is convex.

Now by applying (1.4) for the function $g \circ \phi$, we obtain the following result.

THEOREM 2.6. Let $I, J \subseteq \mathbb{R}$ be intervals and assume that $\phi: I \rightarrow J$ is concave and $g: J \rightarrow \mathbb{R}$ is convex and monotonically decreasing. Then the sequence

$$
a_{n}=n\left[\frac{g\left(\phi\left(x_{1}\right)\right)+\cdots+g\left(\phi\left(x_{n}\right)\right)}{n}-g\left(\phi\left(\frac{x_{1}+\cdots+x_{n}}{n}\right)\right)\right], \quad n \geq 1
$$

is increasing.

First let us put $g(x)=-\ln x$ in (2.2), assuming that $\phi>0$. Hence

$$
\begin{aligned}
a_{n} & =n\left[\ln \phi\left(\frac{x_{1}+\cdots+x_{n}}{n}\right)-\frac{\ln \phi\left(x_{1}\right)+\cdots+\ln \phi\left(x_{n}\right)}{n}\right] \\
& =n \ln \frac{\phi\left(x_{1}+\cdots+x_{n}\right) / n}{\sqrt[n]{\left.\phi\left(x_{1}\right) \cdots+x_{n}\right)}} .
\end{aligned}
$$

Thus we proved the following result.

COROllary 2.7. Let $I \subseteq \mathbb{R}$ be an interval and assume that $\phi: I \rightarrow(0, \infty)$ is concave. Then the sequence

$$
a_{n}=\left[\frac{\phi\left(x_{1}+\cdots+x_{n}\right) / n}{\sqrt[n]{\phi\left(x_{1}\right) \cdots \cdot \phi\left(x_{n}\right)}}\right]^{n}, \quad n \geq 1
$$

is increasing. 
For $p \in \mathbb{R} \backslash\{0\}$ and $x_{1}, \ldots, x_{k}>0$ we put

$$
A_{k}^{p}=\left(\frac{x_{1}^{p}+\cdots+x_{k}^{p}}{k}\right)^{1 / p} .
$$

COROllary 2.8. Let $p \geq 1$ and $x_{1}, \ldots, x_{n}>0$. Then

$$
\left(\frac{A_{n}^{p}}{G_{n}}\right)^{n} \geq\left(\frac{A_{n-1}^{p}}{G_{n-1}}\right)^{n-1} \geq \cdots \geq\left(\frac{A_{1}^{p}}{G_{1}}\right)^{1}=1 .
$$

ProOF. Let us take in (2.3) $\phi(x)=x^{1 / p}$, with $p \geq 1$. Obviously, $\phi$ is concave and (2.3) becomes

$$
a_{n}=\left[\frac{\left(\left(x_{1}+\cdots+x_{n}\right) / n\right)^{1 / p}}{\sqrt[n]{x_{1}^{1 / p} \cdots x_{n}^{1 / p}}}\right]^{n} .
$$

By replacing $x_{k}$ with $x_{k}^{p}$, we obtain that the sequence

$$
a_{n}=\left[\frac{\left(\left(x_{1}^{p}+\cdots+x_{n}^{p}\right) / n\right)^{1 / p}}{\sqrt[n]{x_{1} \cdots x_{n}}}\right]^{n}=\left(\frac{A_{n}^{p}}{G_{n}}\right)^{n}, \quad n \geq 1
$$

is increasing. Hence (2.4) is true.

Finally, note that T. Popoviciu's inequality is the particular case with $p=1$ of (2.4).

\section{References}

[1] D. S. Mitrinović, J. E. Pečarić and A. M. Fink, Classical and new inequalities in analysis (Kluwer Academic Publishers, Dordrecht, 1993).

[2] C. P. Niculescu and L. E. Persson, Convex functions. Basic theory and applications (Universitaria Press, Craiova, 2003). 